\title{
Erratum to: Changes in proteinuria and the risk of myocardial infarction in people with diabetes or pre-diabetes: a prospective cohort study
}

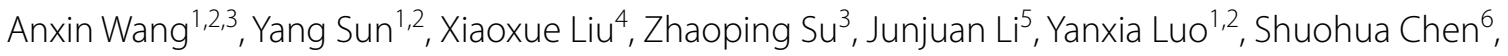
Jianli Wang ${ }^{7}$, Xia Li ${ }^{8}$, Zhan Zhao ${ }^{9,10}$, Huiping Zhu ${ }^{1,2}$, Shouling Wu ${ }^{6^{*}}$ and Xiuhua Guo ${ }^{1,2^{*}}$ (D)

\section{Erratum to: Cardiovasc Diabetol (2017) 16:10 DOI 10.1186/s12933-017-0586-7}

Following publication of the original article [1], the authors reported that figure 2 had not been replaced with the correct figure as indicated in the proofs.

The original article has been corrected.

\section{Publisher's Note \\ Springer Nature remains neutral with regard to jurisdictional claims in pub-} lished maps and institutional affiliations.

Received: 7 September 2017 Accepted: 7 September 2017

Published online: 19 September 2017

\footnotetext{
*Correspondence: drwusl@163.com; statguo@ccmu.edu.cn 1 Department of Epidemiology and Health Statistics, School of Public Health, Capital Medical University, No. 10 Xitoutiao, Youanmenwai, Fengtai District, Beijing 100069, China

${ }^{6}$ Department of Cardiology, Kailuan Hospital, North China University of Science and Technology, No. 57 Xinhua Road, Lubei District, Tangshan 063000, China
}

\author{
Reference \\ 1. Wang A, Sun Y, Liu X, Su Z, Li J, Luo Y, Chen S, Wang J, Li X, Zhao Z, Zhu H, \\ Wu S, Guo X. Changes in proteinuria and the risk of myocardial infarction \\ in people with diabetes or pre-diabetes: a prospective cohort study. \\ Cardiovasc Diabetol. 2017;16:104. doi:10.1186/s12933-017-0586-7.
}

